\title{
MANEJO MÉDICO-NUTRICIONAL DA PANCREATITE AGUDA EM CÃES: RELATO DE CASOS
}

\author{
RONDELLI, M.C.H. ${ }^{1}$ \\ ONDANI, A.C. ${ }^{2}$ \\ TORTOLA, L. ${ }^{3}$ \\ GAVA, F.N. ${ }^{3}$ \\ BORIN, $\mathrm{S}^{3}$ \\ OLIVEIRA, M.C. de C. ${ }^{4}$ \\ CINTRA, T.C.F. ${ }^{3}$ \\ CANOLA, J.C. ${ }^{5}$ \\ CARCIOFI, A.C. ${ }^{5}$ \\ TINUCCI-COSTA, $\mathrm{M}^{5}$
}

Recebido em: 2009-03-29

Aprovado em: 2009-04-29

Issue DOI: $10.3738 / 1982.2278 .189$

RESUMO: A inflamação aguda do pâncreas em cães promove sinais clínicos inespecíficos que incluem êmese e dor abdominal, relacionando-se com fatores de risco tais como dieta com alto teor lipídico, obesidade e idade avançada. Pode conduzir muitos pacientes à morte, em detrimento do avanço das lesões no órgão e estruturas adjacentes, havendo desequilíbrio hidro-eletrolítico severo, comprometimento hepático por lesão direta e renal, por estabelecimento de azotemia grave. O presente trabalho almejou abordar as dificuldades diagnósticas desta enfermidade e a importância do estabelecimento terapêutico rápido, ao relatar dois casos atendidos no Hospital Veterinário da UNESP/Jaboticabal, em um paciente idoso e um outro jovem, que apresentaram manifestação aguda da doença e recuperaram-se satisfatoriamente.

Palavras-chave: Cães. Dor abdominal. Pâncreas.

\section{MEDICAL-NUTRITIONAL MANAGEMENT OF ACUTE Pancreatitis in Dogs: Cases Report}

SUMMARY: The pancreas acute inflammation in dogs provoke non-specific clinical signs that include emesis, abdominal pain and there is a relation with risk factors as feed intake with high levels of lipids, obesity and older age. It may conduct many patients to death due to the advance of lesions in the organ and structures around and hydro-electrolytic disorders, hepatic involvement caused by straight injury and renal consequences, through the

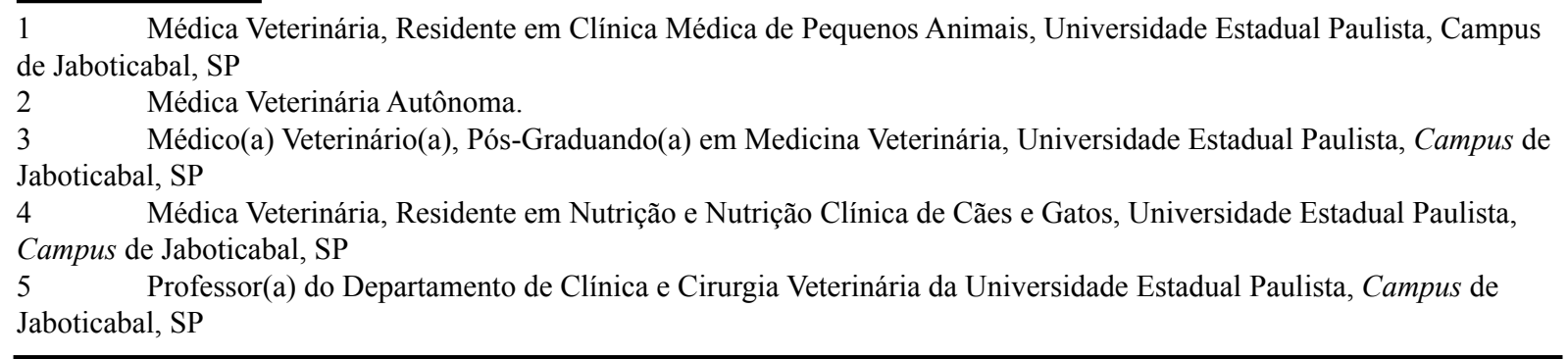

Nucleus Animalium, v.1, n.1, maio 2009 
establishment of severe azotemia. This present paper aimed to report the difficulties in diagnosing this disease and the importance of the quick therapeutic establishment, when it reports two cases assisted at the Veterinary Hospital of UNESP/Jaboticabal, in one elderly patient and other young dog that presented acute manifestation of the disease and recovered in a satisfactory manner.

Keywords: Dogs. Adominal pain. Pancreas.

\section{CONDUCCIÓN MÉDICO-NUTRICIONAL DE LA PANCREATITIS AGUDA EN PERROS: RELATO DE CASOS}

RESUMEN: La inflamación aguda del páncreas en caninos promueve signos clínicos inespecíficos que incluyen emesis y dolor abdominal, relacionados con factores de riesgo tales como la dieta con altos niveles de lípidos, obesidad y edad avanzada. Puede conducir a muchos pacientes a la muerte, por causa del avance de las lesiones en el órgano y en las estructuras adyacentes, produciéndose un desequilibrio hídrico y electrolítico severo, comprometiendo el hígado por una lesión directa y el riñón, por la instauración de azotemia grave. El presente trabajo desea abordar las dificultades diagnosticas de esta enfermedad y la importancia de establecer una terapéutica rápida, relatando dos casos atendidos en el Hospital Veterinario de la UNESP/Jaboticabal, en un paciente geriátrico y en otro joven, que presentaron la manifestación aguda de la enfermedad y se recuperaron satisfactoriamente.

Palabras clave: Perros. Dolor abdominal. Páncreas.

\section{INTRODUÇÃO}

A pancreatite aguda é uma condição inflamatória do pâncreas que ocorre quando as enzimas proteolíticas são ativadas e a auto-digestão do órgão acontece (ELLIOT, 2006). Pode ser classificada como leve (edematosa) ou intensa (hemorrágica) (SHERDING; BIRCHARD; JOHNSON apud BIRCHARD; SHERDING, 2003). É uma enfermidade clinicamente importante em cães, de alta mortalidade, especialmente quando concomitante com outras patologias. Seu diagnóstico precoce, assim como a rápida instituição de terapia são importantes para a recuperação do animal (WATSON, 2007a).

A etiologia e os mecanismos de estabelecimento da pancreatite estão pouco elucidados. Uma variedade de aspectos pode contribuir para que a doença se manifeste sendo, a obesidade, a ingestão de dietas ricas em lipídios, a hiperlipidemia, endocrinopatias, administração de drogas e ingestão de toxinas considerados importantes fatores de risco (SIMPSON, 2004). Entretanto, 90\% das pancreatites agudas são julgadas idiopáticas (WATSON, 2007a).

O diagnóstico da pancreatite aguda é baseado na combinação de informações clínicas, clínico-patológicas e achados de imagem (SIMPSON, 2004).

O propósito do texto em tela é ilustrar o sucesso obtido na abordagem diagnóstica e terapêutica de dois casos atendidos no Hospital Veterinário da UNESP/Jaboticabal, quando foram empregados os manejos médicos e nutricionais para a recuperação dos pacientes. 


\section{RELATOS DE CASOS}

\section{Caso 1}

Foi atendida, no Hospital Veterinário da UNESP/Jaboticabal, uma cadela sem definição racial, de 11 anos, com 17,7 kg de massa corpórea cuja queixa era a ocorrência de dez episódios eméticos durante o dia anterior ao atendimento. $\mathrm{O}$ animal havia ingerido carne bovina condimentada e gordurosa antes do início dos sinais clínicos mencionados. Ao exame físico, não foram observadas alterações importantes, exceto dor à palpação abdominal.

Dentre os exames complementares realizados, apresentaram-se aumentadas apenas as concentrações da alanina amino-transferase e fosfatase alcalina; a amilase e lipase estavam dentro da normalidade. Radiograficamente observou-se aumento de radiopacidade de tecidos moles com perda parcial de detalhes da serosa das vísceras em região abdominal cranial, sugerindo uma peritonite focal. Observou-se deslocamento do duodeno para a direita, espessamento da parede duodenal e padrão de gás estático. Ao exame ultra-sonográfico, o lobo pancreático direito apresentou dimensões aumentadas e ecogenicidade reduzida. $\mathrm{O}$ mesentério circunjacente apresentou-se hiperecogênico e de aspecto sólido. $\mathrm{O}$ animal manifestou desconforto com a pressão do transdutor durante o exame. As alterações observadas sugeriram ocorrência de pancreatite de curso agudo com peritonite focal associada.

Imediatamente, instituiu-se fluidoterapia por via intravenosa com Ringer com Lactato de Sódio, associada a administração de Cloridrato de Metoclopramida, Cloridrato de Ranitidina, Cloridrato de Tramadol e Sulfato de Atropina, por via subcutânea. Foram eleitos para terapia antimicrobiana os fármacos Sulfametoxazol associado ao Trimetoprim, administrados por quinze dias por via subcutânea e Metronidazol por seis dias, por via intravenosa. O animal permaneceu hospitalizado por quatro dias consecutivos, em condições de jejum hídrico e alimentar, instituídas como tratamento nutricional no primeiro dia de internamento. A partir do segundo dia, utilizou-se durante dois dias, a nutrição parenteral parcial a fim de suprir parte das necessidades energéticas, composta basicamente de aminoácidos, glicose e eletrólitos via cateter venoso periférico, mantendo o jejum hídrico e alimentar. No terceiro dia, iniciou-se a nutrição microenteral, com composição semelhante a da nutrição parenteral, na dose inicial de $0,05 \mathrm{~mL} / \mathrm{kg} /$ hora em bólus, aumentando-se gradativamente até $1,0 \mathrm{~mL} / \mathrm{kg} /$ hora e administração restrita de água por via oral. No quarto dia, uma dieta enteral com baixo teor lipídico (5\%), utilizando ingredientes como dextrose, farinha de arroz, extrato de soja e suplemento mineral e vitamínico, suprindo somente $35 \%$ das necessidades energéticas de manutenção, dividida em quatro porções ao longo do dia, foi instituída. No quinto dia, o animal já apresentava melhora significativa e ausência de vômitos, decidindo-se pela alta médica. Como tratamento em domicílio, prescreveu-se uma dieta caseira com baixo teor lipídico (5\%), utilizando-se arroz, peito de frango, cenoura e suplemento mineral e vitamínico, aumentando sua quantidade gradativamente até atingir as necessidades energéticas de manutenção (NRC, 2006).

No retorno, após sete dias, o ultra-som ainda mostrava áreas reativas no pâncreas e 
peritonite focal na exposição radiográfica, embora clinicamente o animal estivesse bem. Foi sugerida a redução de peso corpóreo em $15 \%$ e dieta super premium light para atender a $75 \%$ das necessidades.

\section{Caso 2}

Uma cadela Labrador, de 3 meses, 9,2 kg de massa corporal, foi atendida no Hospital Veterinário da UNESP/Jaboticabal, com a queixa de vários episódios eméticos em um dia. $\mathrm{O}$ animal já havia recebido duas doses de vacina óctupla e vermífugo e era mantido em domicílio sem acesso à rua. Alimentava-se exclusivamente de ração econômica para filhotes e apresentava apetite depravado. Ao exame físico, observaram-se parâmetros fisiológicos dentro da normalidade, porém encontrava-se apático e apresentava dor à palpação abdominal.

$\mathrm{O}$ hemograma revelou leve anemia e leucocitose com desvio à esquerda. Os valores de amilase e lipase encontravam-se sem alteração. À radiografia abdominal, pôde-se observar aspecto de peritonite, sem evidências de corpo estranho radiopaco. $\mathrm{O}$ duodeno apresentouse deslocado para a direita, com padrão de gás estático e aparência de letra " $C$ ". Ao exame ultra-sonográfico, o lobo pancreático esquerdo apresentou dimensões aumentadas, superfície irregular e padrão de ecogenicidade misto, demonstrando aspecto heterogêneo. O mesentério circunjacente apresentou-se hiperecogênico e o animal demonstrou desconforto à passagem do transdutor sobre a região, sugerindo a ocorrência de pancreatite.

O paciente foi hospitalizado e aos moldes do relato anterior, utilizou-se solução de Ringer com Lactato de Sódio, por via intravenosa durante quatro dias consecutivos e terapia medicamentosa com Cloridrato de Ranitidina, Cloridrato de Metoclopramida, Cloridrato de Tramadol e Ampicilina Sódica. Por via intravenosa, Metronidazol foi administrado por três dias. Para suprir as necessidades energéticas de manutenção, também empregou-se, a partir do segundo dia, nutrição parenteral parcial. No quarto dia de tratamento, introduziu-se, gradativamente, dieta microenteral e ingestão hídrica restrita, como no relato anterior, que foi bem aceita pelo animal. Neste momento, o hemograma apresentava redução da leucocitose, já sem desvio. A amilase e lipase permaneceram dentro dos limites de normalidade. Nesse mesmo dia, iniciou-se a medicação por via oral (Cloridrato de Ranitidina e Ampicilina por mais seis dias, e Metronidazol por mais três dias). No dia seguinte, iniciou-se a alimentação enteral utilizando-se dieta com baixo teor lipídico (5\%), seguido de uma dieta caseira semelhante a do caso anterior, com início gradual.

No retorno, em sete dias, o animal encontrava-se saudável e já não se observaram alterações hematológicas. A imagem ultra-sonográfica do pâncreas mostrou discreta redução das dimensões, mantendo ainda o padrão de ecogenicidade mista e aspecto heterogêneo em comparação com o primeiro exame. Quanto à alimentação, foi prescrita ração super premium para filhotes, com quantidade calculada a partir das necessidades energéticas de crescimento, segundo NRC (2006). 


\section{DISCUSSÃO}

Suspeita-se de pancreatite, quando se observa surgimento agudo de êmese acompanhada por dor abdominal (SIMPSON, 2004). Entre os fatores de risco figuram a idade, obesidade e a ingestão de dietas ricas em lipídios (SIMPSON, 2004), coincidentes com o perfil do animal do primeiro caso.

A ocorrência de pancreatite é vastamente descrita pela literatura em cães adultos e idosos (BUNCH, 2006 apud NELSON; COUTO; CULLEN, 2007; CULLEN apud MCGAVIN; ZACHARY, 2007), contudo o acometimento de filhotes de cães não é comumente documentado.

A pancreatite aguda em cães tipicamente envolve o fígado devido à proximidade entre os órgãos, permitindo a direta extensão da inflamação (BUNCH, 2006 apud NELSON; COUTO; CULLEN, 2007), justificando, em muitos casos, o aumento nos valores de enzimas hepáticas.

A mensuração das enzimas amilase e lipase são consideradas como método diagnóstico de pancreatite, apesar de apresentar especificidade de 50\% (TWEDT, 2007), pois podem estar aumentadas em casos de enfermidades não-pancreáticas, uma vez que são secretadas em diferentes sítios (BUNCH, 2006 apud NELSON; COUTO; CULLEN, 2007), como também, podem não se alterar em enfermidades pancreáticas (SIMPSON, 2004).

O exame radiográfico é considerado inespecífico na pancreatite aguda, mas pode revelar aumento da opacidade no quadrante direito cranial abdominal, duodeno dilatado e deslocado ventralmente ou para a direita (Fig. 1) (SIMPSON, 2004), tal como neste relato, indicando a reação local que ocorre, uma vez que há a liberação de enzimas pancreáticas ativadas e fatores mediadores inflamatórios (SIMPSON, 2004). O exame ultra-sonográfico é útil no diagnóstico da pancreatite, demonstrando edema e peritonite focal (WATSON, 2007a), como observados nos casos supra-descritos.

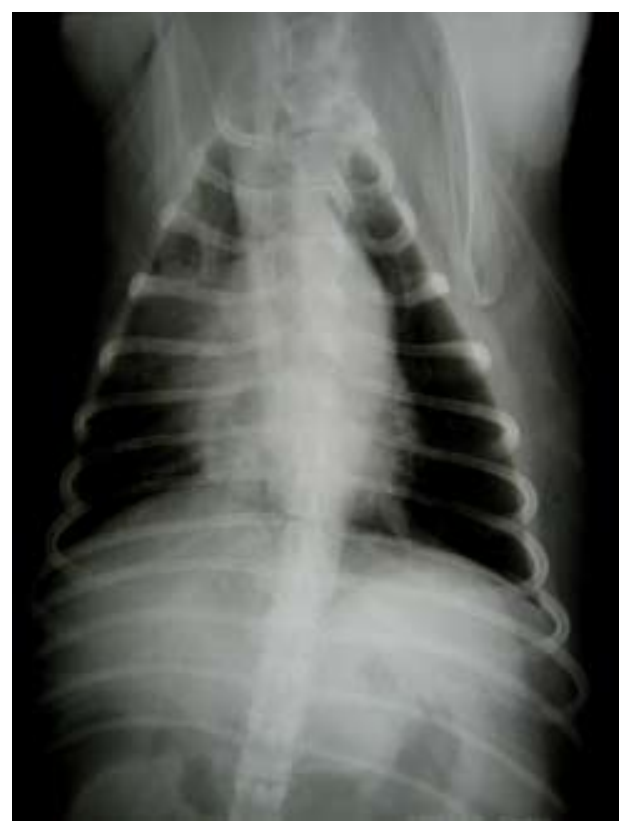

FIGURA 1- Imagem radiográfica em posição ventro-lateral, de um cão acometido por pancreatite aguda. Notar o duodeno deslocado cranialmente, em posição de "C" (setas) e o aspecto de peritonite focal adjacente. 
A utilização de um anti-emético, associado a um inibidor da liberação de secreção ácida estomacal (Cloridrato de Metoclopramida e Ranitidina, respectivamente) é de fundamental importância no tratamento, reduzindo o vômito e diminuindo a gastrite causada pelo mesmo. No primeiro caso, na tentativa de se reduzir a salivação excessiva, foi administrado Sulfato de Atropina, devido sua ação anti-muscarínica atuando na redução da secreção salivar (VITAL, 2002). A promoção de analgesia é preconizada por vários autores (SIMPSON, 2004; WATSON 2007a) e foi conseguida com a administração de Cloridrato de Tramadol. Os distúrbios eletrolíticos são alterações comuns na pancreatite aguda, em conseqüência da maior perda de eletrólitos nos casos de vômitos ou diarréia, menor ingestão destes íons ou por desvios celulares (SIMPSON; BIRNBAUM, 2007). Desta forma, recomenda-se o uso de fluidoterapia repositora de eletrólitos, sendo o Ringer com Lactato de Sódio o fluido de escolha para esses casos. Embora as bactérias não desempenhem um papel primário na pancreatite aguda canina, o pâncreas necrótico é um bom meio de cultura para o crescimento bacteriano. Assim sendo, recomendase a administração de antimicrobianos sistêmicos, tais como Sulfametoxazol-Trimetoprim e a Ampicilina (BUNCH, 2006 apud NELSON; COUTO; CULLEN, 2007). A associação, em ambos os casos, de um antimicrobiano ao Metronidazol, objetivou aumentar o espectro de ação e reduzir a inflamação (WATSON, 2007b).

A hospitalização é de fundamental importância, uma vez que o animal deve ser submetido a jejum hídrico e alimentar e deve ter suas necessidades hídricas e nutricionais supridas por via parenteral, em uma tentativa de coibir estímulos ao vômito e à liberação de enzimas digestivas. Embora, na visão de alguns autores, o uso da nutrição enteral jejunal sobre a parenteral ofereça melhor resultado para o paciente com pancreatite aguda (QIN et al., 2007; MAKOLA et al., 2007), nos casos acima relatados, optou-se pelo uso de nutrição parenteral parcial devido à impossibilidade da realização de jejunostomia ou da colocação de sonda nasojejunal por meio de endoscopia. Para compensar os possíveis efeitos deletérios do não uso do trato gastrointestinal, utilizou-se a fluidoterapia microenteral, proposta por Crowe (1989), a qual consiste em administrar pequenas quantidades de água, eletrólitos e nutrientes facilmente absorvíveis (glicose, aminoácidos e pequenos peptídeos), por via oral, em bólus ou infusão constante.

A terapia nutricional parenteral (Fig. 2, 3 e 4) consiste na administração das exigências nutricionais diárias do animal por via intravenosa. Em ambos os casos ora relatados, utilizou-se a nutrição parenteral parcial por meio de cateter intravenoso periférico sem adição de lipídios na formulação devido à indisponibilidade deste, embora sua utilização por via parenteral na pancreatite aguda não estimule a liberação de secreção pancreática, podendo, portanto, ser utilizada (STABILE et al., 1984), diferentemente da via enteral, na qual a ingestão de lipídios estimula a secreção das enzimas pancreáticas. Consequentemente, optou-se por uma dieta enteral com baixo teor lipídico, semelhante ao proposto por Jacobson et al. (2007). 


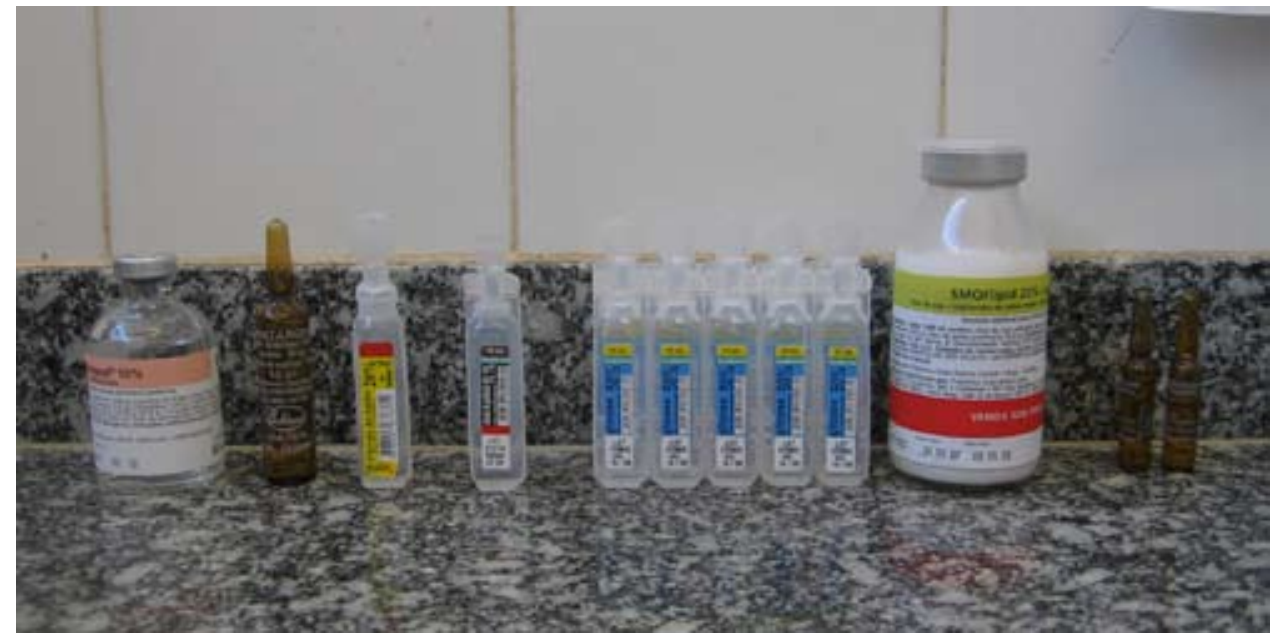

FIGURA. 2 - Alguns componentes da Nutrição Parenteral.

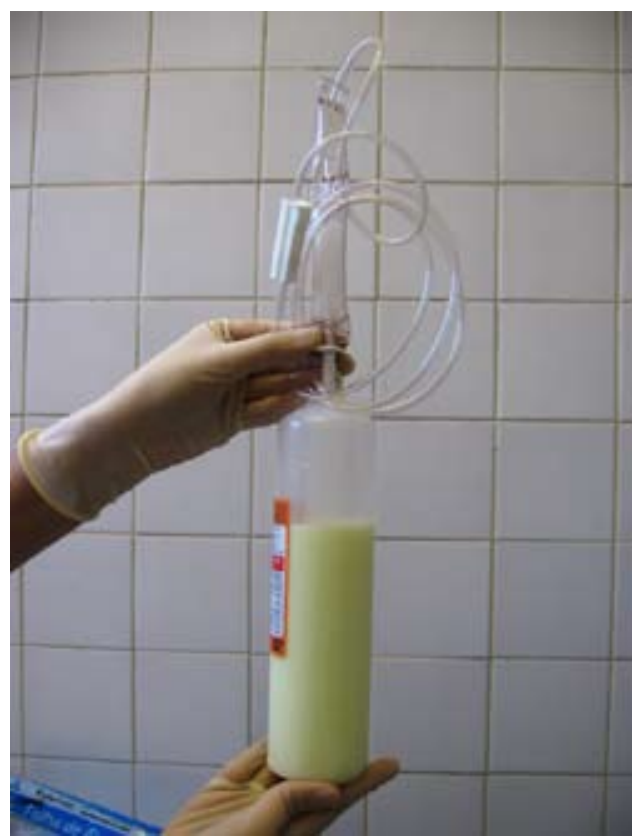

FIGURA.3 Nutrição Parenteral preparada

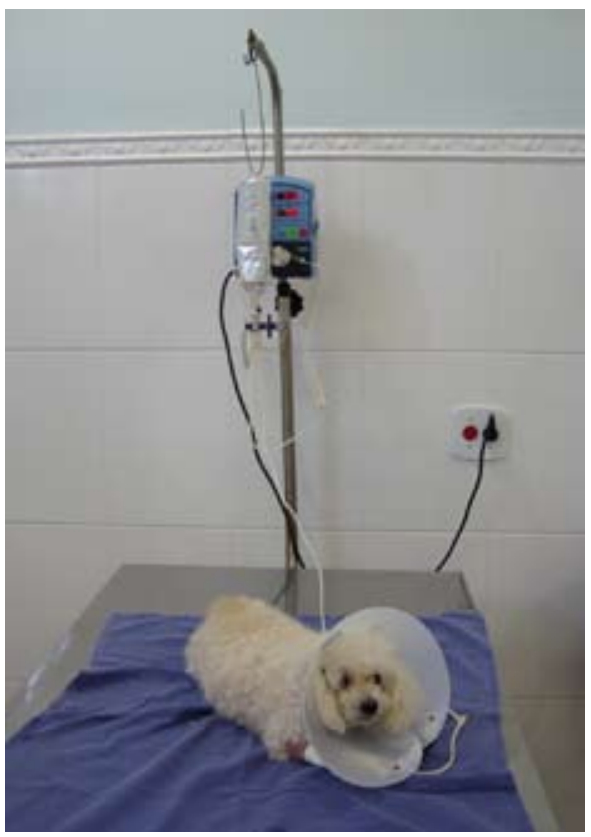

FIGURA.4 Paciente recebendo terapia nutricional parental

A adoção imediata de um tratamento medicamentoso e nutricional planejados em casos de pancreatite aguda melhora o prognóstico e pode garantir a recuperação dos animais.

\section{CONCLUSÃO}

A pancreatite aguda em cães representa um desafio diagnóstico e terapêutico na clínica de pequenos animais. Os sinais clínicos manifestados são, por vezes, inespecíficos e requerem do clínico a perspicácia de avaliar o histórico do animal e conciliá-lo com aspectos encontrados nos exames complementares, essencialmente nos achados ultra-sonográficos referentes ao pâncreas edematoso e à peritonite focal. 
A intervenção clínica deve ser imediata, adotando-se medidas médicas e nutricionais que visem a retardar a progressão da lesão ao órgão e estruturas adjacentes. Isso inclui, em linhas gerais, modular a produção enzimática pancreática, para que se procurava um "repouso" necessário ao órgão. Tal meta é alcançada por meio do estabelecimento de jejum hídrico e alimentar e suplementação nutricional adequada do paciente com métodos como dieta parenteral seguida de dieta enteral balanceada, além de correção do desequilíbrio hidro-eletrolítico, instituição de terapia visando, essencialmente, ao controle emético, à analgesia adequada e à adoção de antibióticos direcionados à peritonite focal.

\section{REFERÊNCIAS}

BUNCH, S.E. O pâncreas exócrino, p.533-546. In: NELSON R.W. ; COUTO C.G. Medicina interna de pequenos animais. 3.ed. São Paulo: Elsevier, 2006.

CULLEN, J.M. Liver, biliary system, and exocrine pancreas. In: MCGAVIN, M.D.; ZACHAR, J.F. Pathologic basis of veterinary disease. 4 ed. St. Louis: Elsevier, 2007. p. 393 - 461.

ELLIOT, D. Nutritional management of canine pancreatitis. WSAVA 2006. Disponível em: http://www.ivis.org. Acesso em: 20 maio 2008.

JACOBSON, B.C.et al. A prospective, randomized trial of clear liquids vs. low-fat solid diet as the initial meal in mild acute pancreatitis. Clin Gastroenterol Hepatol. v.5, n.8, p.946-951, 2007.

MAKOLA, D.; KRENITSKY, J.; PARRISH, C.R. Enteral feeding in acute and chronic pancreatitis. Gastrointest Endoscopy Clin N Am. n.17, p.747-764, 2007.

NRC-National Research Council. Nutrient requirements of dogs. Washington: National Academy Press,2006. p.398.

QIN, H.L. et al. Effect of parenteral and early intrajejunal nutrition on pancreatic digestive enzyme synthesis, storage and discharge in dog models of acute pancreatitis. World Journal of Gastroenterology, v.13, n.7, p.1123-1128, 2007.

SHERDING R.G.; BIRCHARD S.J.; JOHNSON S.E. Doenças e cirurgia do pâncreas exócrino. In: BIRCHARD S.J.; SHERDING R.G. Clínica de pequenos animais. São Paulo: Roca, 2003. p. 965 - 974. (Manual Saunders) 
SIMPSON, K.W.; BIRNBAUM, N. Distúrbios hidroeletrolíticos nas doenças pancreáticas e gastrintestinais. In: DiBARTOLA, S.P. Anormalidades de fluidos, eletrólitos e equilíbrio ácido-básico na clínica de pequenos animais. 3.ed. São Paulo: Roca, 2007. p.405-420.

SIMPSON, K.W. Acute pancreatitis. In: CANINE and feline nutrition: esource for Companion Animal Professionals. 2.ed. St. Louis: Mosby, 2004. p. 186-196.

STABILE, B.E.et al. Intravenous mixed amino acids and fats do not stimulate exocrine pancreatic secretion. Am J Physiol Gastrointest Liver Physiol. v.246, n.3, p.274-G280, 1984.

TWEDT, D. Diagnosis and treatment of pancreatitis. South European Veterinary Conference. 2007. Disponível em: http://www.ivis.org. Acesso em: 20 abr. 2008.

WATSON, P. Acute pancreatitis in dogs. The North American Veterinary Conference. 2007ª. Disponível em: http://www.ivis.org. Acesso em: 15 maio 2008.

WATSON P. Chronic pancreatitis: an underestimated disease. The North American Veterinary Conference. Disponível em: http://www.ivis.org. Acesso em: 13 maio 2008. 2007b.

VITAL, M.A.B.F. Agonistas e antagonistas colinérgicos. In: SPINOSA H.S., GÓRNIAK S.L.; BERNARDI M.M. (Ed.). Farmacologia aplicada à medicina veterinária. 3.ed. São Paulo: Guanabara Koogan, 2002., p.59- 71. 